\title{
Tailor-made virtual lung: prevailing clinical application
}

\author{
Tadashi Akiba, MD
}

Received: 21 December 2008

(C) The Japanese Association for Thoracic Surgery 2009

Key words Double-lumen endotracheal tube $\cdot$ Threedimensional multidetector computed tomography · Virtual lung $\cdot$ Video-assisted thoracic surgery

Computed tomography (CT) can provide detailed twodimensional images of the bronchial branching pattern and pulmonary vessels; however, it is sometimes difficult to grasp the three-dimensional (3D) images. CT scan technology has advanced from conventional and spiral $\mathrm{CT}$ to multidetector (MD) CT, and we can now obtain 3D images of pulmonary structures with little effort. Physicians can use 3D-MDCT images to view more detailed regional anatomy of individual patients. For the bronchi and pulmonary vessels, for instance, 3D-MDCT can show the length or diameter of the branches, and the angles and the distance between the branches. Such information can aid physicians in better understanding a lesion and performing various medical interventions.

Okuda et al. measured the right main bronchial diameters using chest MDCT to select the appropriate rightsided double-lumen endotracheal tube (DLT). Although there are many variations of tracheobronchial trees, 3DMDCT can show the inner shape of the right main bronchi in detail. This study is important and interesting

This editorial refers to the article by Okuda et al. on pp. 369-375 of this issue of General Thoracic and Cardiovascular Surgery.

T. Akiba $(\bowtie)$

Department of Surgery, Jikei University Kashiwa Hospital,

163-1 Kashiwashita, Kashiwa, Chiba 277-8567, Japan

Tel. +81-4-7164-1111; Fax +81-4-7163-3488

e-mail: akiba@jikei.ac.jp because it presents basic data of the right main bronchi of living patients for the purpose of safe one-lung ventilation using right-sided DLT.

An appreciation of the anatomical advantage of the longer left main bronchus has directed anesthetists to prefer left-sided DLT, if possible, for lung separation. In addition, they also prefer a bronchial blocker tube, especially for patients with airway abnormalities or when the surgeon requests no tube on the left side. ${ }^{1,2}$ There are reports in which the DLT was chosen by measuring the size of the left main bronchi using MDCT. ${ }^{3}$ On the other hand, there are few reports that have investigated the right main bronchi using right-sided DLT because its application is relatively rare.

The size and shape of tracheobronchial trees detected as images using 3D-MDCT can change owing to many factors, including airway pressure, pressure of a pleural cavity, and compression of surrounding organs. Furthermore, the cross section of the right main bronchi is not round. Therefore, it may be necessary to measure at least the mediolateral and anteroposterior bronchial diameters on the proximal and distal sides to know the true shape of the right main bronchi. Okuda et al., however, measured only proximal mediolateral diameters and the length of the right main bronchi. Furthermore, no data were presented on the use of right-sided DLT in the patients. Thus, it could not be determined whether they were able to apply correctly the results of the 3D-MDCT measurements.

Okuda et al. reported that as many as one-third of the patients (34/100 patients) had problems that required attention during right-sided bronchial tube insertion, and 31 of the 34 problematic patients had bronchi $<10 \mathrm{~mm}$ diameter in the right main bronchi. Their Fig. 6, however, shows no patients with a diameter 


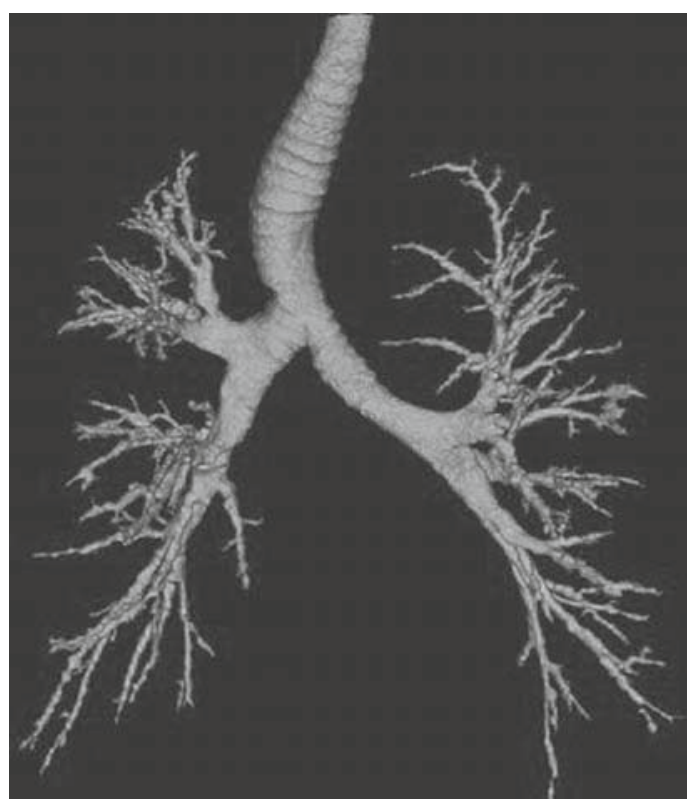

Fig. 1 Tracheobronchial tree using multidetector computed tomography (MDCT)

$<10 \mathrm{~mm}$. They also reported that 11 of the 34 patients who had problems had bronchi $<5 \mathrm{~mm}$ in length. In the abstract, however, they concluded that patients with bronchial lengths $<10 \mathrm{~mm}$ were problematic. The critical size could not be evaluated from the data presented in this article.

MDCT enables detailed, individual images of the chest anatomy of patients displaying the tracheobronchial tree, pulmonary vessels, and coronary arteries. One of the important merits of 3D-MDCT is that it shortens the reading time of the images and decreases the chance of missing variations in anatomy.

Virtual bronchoscopy produces images of the tracheobronchial tree, and the endobronchial views simulate the findings of conventional bronchoscopy. ${ }^{4}$ For living donor lung transplantation, 3D-MDCT angiography has been used to show the lung anatomy of the proposed donors to select a donor lung. ${ }^{5}$ For lung cancer surgery, especially video-assisted thoracic surgery, preoperative 3D-MDCT angiography has been used to visualize the pulmonary arteries, which are important factors in lung resection. ${ }^{6-8}$ Variations of the pulmonary veins in the left atrium have been thoroughly described using 3D-CT for treating patients with atrial fibrillation. ${ }^{9}$ In that study, $71 \%$ of the patients had two ostia on the right side, $28 \%$ had three to five ostia, $86 \%$ had two ostia on the left side, and the remainder (14\%) had a single ostium.

At Jikei University Kashiwa Hospital, patients who are candidates for general thoracic surgeries are rou-

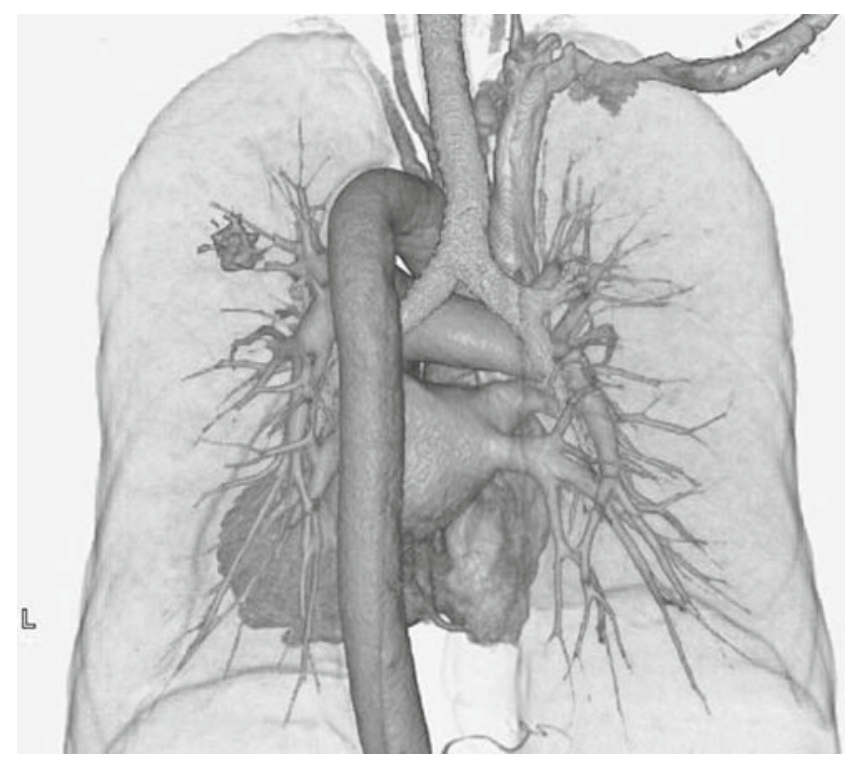

Fig. 2 "Tailor-made virtual lung." Tracheobronchial tree and pulmonary vessels are shown

tinely examined by 64-row 3D-MDCT angiography and virtual bronchography with iodinated contrast medium. ${ }^{7,8}$ Radiology technicians obtain images of the bilateral lung, pulmonary vessels, tracheobronchial trees, and abnormal lesions (Fig. 1). A "tailor-made virtual lung," constructed with these 3D images (Fig. 2), contribute to preoperative evaluation.

\section{References}

1. Russell WJ. A logical approach to the selection and insertion of double-lumen tubes. Curr Opin Anaesthesiol 2008; 21:37-40.

2. Campos JH. Which device should be considered the best for lung isolation: double-lumen endotracheal tube versus bronchial blockers. Curr Opin Anaesthesiol 2007;20:27-31.

3. Jeon Y, Ryu HG, Bahk JH, Jung CW, Goo JM. A new technique to determine the size of double-lumen endobronchial tubes by the two perpendicularly measured bronchial diameters. Anaesth Intensive Care 2005;33:59-63.

4. Horton KM, Horton MR, Fishman EK. Advanced visualization of airways with 64-MDCT: 3D mapping and virtual bronchoscopy. AJR Am J Roentgenol 2007;189:1387-96.

5. Duong PA, Ferson PF, Fuhrman CR, McCurry KR, Lacomis JM. 3D-multidetector CT angiography in the evaluation of potential donors for living donor lung transplantation. J Thorac Imaging 2005;20:17-23.

6. Watanabe S, Arai K, Watanabe T, Koda W, Urayama H. Use of three-dimensional computed tomographic angiography of pulmonary vessels for lung resections. Ann Thorac Surg 2003;75:388-92.

7. Akiba T, Marushima H, Takagi M, Odaka M, Harada J, Kobayashi S, et al. Preoperative evaluation of a tracheal bronchus by three-dimensional 64-row multidetector-row computed tomography (MDCT) bronchography and angiography: report of a case. Surg Today 2008;38:841-3. 
8. Akiba T, Marushima H, Harada J, Kobayashi S, Morikawa T. Anomalous pulmonary vein detected using three-dimensional computed tomography in a patient with lung cancer undergoing thoracoscopic lobectomy. Gen Thorac Cardiovasc Surg 2008;56:413-6.
9. Marom EM, Herndon JE, Kim YH, McAdams HP. Variations in pulmonary venous drainage to the left atrium: implications for radiofrequency ablation. Radiology 2004;230:824-9. 